\title{
Durable and Superhydrophobic Aluminium Alloy with Microscale Hierarchical Structures and Anti-Drag Function Inspired by Diving Bell Spider
}

\author{
You Chen ${ }^{1}$, Zijing Quan ${ }^{1}$, Yuhan Sun ${ }^{1}$, Deqiang Chi ${ }^{1}$, Delei Liu ${ }^{1}$, Liang Zhou ${ }^{1}$, Junqiu Zhang ${ }^{1}$, Zhengzhi Mu ${ }^{1}$, \\ Ze Wang ${ }^{1}$, Bo Li ${ }^{1,2, *(\mathbb{D})}$, Shichao Niu ${ }^{1, * \mathbb{D}}$, Zhiwu Han ${ }^{1}$ and Luquan Ren ${ }^{1}$ \\ 1 Key Laboratory of Bionic Engineering, Ministry of Education, Jilin University, Changchun 130022, China; \\ chenyou19@mails.jlu.edu.cn (Y.C.); quanzj19@mails.jlu.edu.cn (Z.Q.); sunyh1818@mails.jlu.edu.cn (Y.S.); \\ chidq19@mails.jlu.edu.cn (D.C.); ld120@mails.jlu.edu.cn (D.L.); liangzhou19@mails.jlu.edu.cn (L.Z.); \\ junqiuzhang@jlu.edu.cn (J.Z.); zmu@jlu.edu.cn (Z.M.); wangze@jlu.edu.cn (Z.W.); zwhan@jlu.edu.cn (Z.H.); \\ lqren@jlu.edu.cn (L.R.) \\ 2 Department of Chemistry, University of California, Riverside, CA 92521, USA \\ * Correspondence: boli@jlu.edu.cn (B.L.); niushichao@jlu.edu.cn (S.N.)
}

check for updates

Citation: Chen, Y.; Quan, Z.; Sun, Y.; Chi, D.; Liu, D.; Zhou, L.; Zhang, J.; Mu, Z.; Wang, Z.; Li, B.; et al. Durable and Superhydrophobic Aluminium Alloy with Microscale Hierarchical Structures and Anti-Drag Function Inspired by Diving Bell Spider. Coatings 2021, 11, 1146. https:// doi.org/10.3390/coatings11101146

Received: 24 August 2021

Accepted: 16 September 2021

Published: 22 September 2021

Publisher's Note: MDPI stays neutral with regard to jurisdictional claims in published maps and institutional affiliations.

Copyright: (c) 2021 by the authors. Licensee MDPI, Basel, Switzerland. This article is an open access article distributed under the terms and conditions of the Creative Commons Attribution (CC BY) license (https:// creativecommons.org/licenses/by/ $4.0 /)$

\begin{abstract}
Coating materials with special surface wettability are widely applied in marine paint systems used in the naval industry to reduce the corrosion and viscous drag of seawater. However, traditional coatings are inefficient and limited, either by poor durability or insufficient anti-drag capacity. Here, inspired by the diving bell spider, a bionic superhydrophobic coating with multiscale hierarchical architecture was successfully prepared on the surface of aluminium alloy. It possesses excellent mechanical abrasion durability, chemical durability, and low adhesion. Remarkably, the water contact angles could remain over $150.9^{\circ}$ after more than 15 abrasion cycles or strong acid/alkali conditions. In addition, the impacting water droplet lifted off the surface of bionic superhydrophobic aluminium alloy (BSAA) within $13 \mathrm{~ms}$, illustrating an excellent low adhesion property. In fact, when the BSAA is immersed in water, it could absorb bubbles and form a gas membrane. The existence of the gas membrane could prevent water and anaerobic organisms from contacting and even corroding the BSAA. Meanwhile, the gas membrane acts as a lubricant and significantly deceases friction at the solid-liquid interface, reducing the drag for BSAA. The BSAA proposed in this work has broad application prospects, such as medical devices, microfluidic chips, gas separation and collection in water.
\end{abstract}

Keywords: bio-inspired; diving bell spider; superhydrophobic aluminium alloy; physicochemical durability; anti-drag function

\section{Introduction}

In the past few decades, to maximize the energy efficiency (like increasing the traveling speed and distance of underwater naval vehicles), and minimize the drag losses in fluid transport in pipelines, scientists have made gigantic efforts to develop strategies to reduce the corrosion and viscous drag of seawater [1-3], which accounts for a relatively high proportion of the total dissipation of well-designed underwater vehicles. To avoid the adhesion of aquatic organisms and the corrosion of seawater, marine vehicles are generally covered by superhydrophobic paint [4-8]. Generally, when the contact angle of the surface is greater than $90^{\circ}$, it is defined as a hydrophobic surface. In particular, when the contact angle is greater than $150^{\circ}$ and the sliding angle (SA) is less than $10^{\circ}$, water droplets could maintain a spherical shape on the solid surface, which is called a superhydrophobic surface [9-12]. However, the conventional superhydrophobic paint focuses on enhancing the water-repellent performance solely, which neglects the physicochemical durability [6,13-15]. Therefore, superhydrophobic coating materials [16-20] are prone to be decimated or even fall off from the substrates [21], resulting in the invalidation of 
the water-repellent function, especially for marine vehicles working in harsh environments, such as deep sea and polar regions. Considering the above-mentioned challenges, the water-repellent function should be achieved by a structural design to replace traditional superhydrophobic paint [22-25], enhance the physicochemical strength, and further synergistically improve the anti-drag performance of superhydrophobic coating materials, which is in line with the developmental tendencies of superhydrophobic coating materials [26-29].

The effective integration of multiple properties into one material remains a tremendous challenge, which requires novel designs and thought-provoking mechanisms [30-33]. For structural design, as we all know, after millions of years of brutal evolution, creatures in nature have evolved diversified micro/nanostructures to realize a great number of optimized functions for adapting to varied environmental changes [34-37]. These biological materials with functional integration provide an enormous source of inspiration for the development of biomimetic materials [38,39].

The palearctic diving bell spider Argyroneta aquatica is a unique spider that lives and hunts among water plants in ponds, ditches, and lakes. Fine hydrophobic structures on the abdomen hold a thin layer of air, which enables the spider to breathe with its book lungs and tracheal system under water $[40,41]$. The shape of the abdomen is similar to that of rugby. Furthermore, the abdomen's surface is completely covered by countless loosely stacked waterproof bristles. The base of the spider's abdomen is distributed with corrugated groove-shaped microstructures, and a single bristle is embedded in a "socket" similar to a round hole, which plays a certain role in securing the bristles. However, the underlying mechanism is neither very clear nor unified. Moreover, no one has applied this structure to capture bubbles underwater so far [20,42].

Inspired by the multiscale hierarchical structure of the diving bell spider, a bionic superhydrophobic aluminium alloy (BSAA) with excellent mechanical abrasion durability, superior chemical durability, and low adhesion properties was prepared using the method of combing the spray coating and laser corrosion. Afterwards, the element distribution and morphology of the BSAA were studied with the help of an Energy Dispersive Spectrometer (EDS) and Scanning Electron Microscope (SEM). Then, the characteristics of mechanical abrasion durability, chemical durability, and adhesion were carried out systematically. The bubble capture capability of BSAA was proven by a self-built high-speed camera system. What is more, a set of optimized models were designed, and the nonwetting mechanism of the BSAA was investigated experimentally. In fact, when the BSAA was dipped in water, it could attract bubbles in the water and form a gas membrane. The gas membrane could prevent the water and anaerobic organisms from contacting and even corroding the BSAA. At the same time, the gas membrane could also significantly decease friction at a solid-liquid interface, reducing the adhesion and drag of water and anaerobic organisms for BSAA. The investigations in this work offer a promising way to handily design and fabricate quasi-textured surfaces with multiscale hierarchical structures that possess excellent durability and superior water-repellent capacity.

\section{Materials and Methods}

\subsection{Materials}

$\mathrm{TiO}_{2} \mathrm{MPs}$ (ca, $0.3 \mu \mathrm{m}$ in diameter) were purchased from Shanghai Aladdin Biochemical Technology Co., Ltd., Shanghai, China. 30 wt.\% ammonium hydroxides were purchased from Tianjin Fuyu Fine Chemical Co., Ltd., Tianjin, China. Hexamethyldisilane (HMDS) was obtained from Aladdin Reagent Co., Ltd., Shanghai, China. Tetraethyl orthosilicate was purchased from Shanghai Aladdin Biochemical Technology Co., Ltd., Shanghai, China. Absolute ethanol (99.5\%) was obtained from Beijing Chemical Works, Beijing, China. All of the chemical reagents were of analytical grade and were used without further treatment. All the aluminium alloys used in this work were purchased from Shenzhen Manlong Metal Products Co., Ltd., Shenzhen, China. 


\subsection{Preparation of Aluminium Alloy Substrates}

The aluminium substrate, which had a geometry of $45 \times 85 \times 1 \mathrm{~mm}^{3}$, was ablated point by point by a picosecond pulse (PSP) laser (FB20-SBGZ, JonteLaser, Beijing, China). The microstructure was obtained with a diameter of $100 \mu \mathrm{m}$, a depth of $60 \mu \mathrm{m}$, and a spacing of $100 \mu \mathrm{m}$. The laser-processed surface was immersed in a water bath for heating. This stabilized the structure shape and size of the sample. Hence, a more obvious micro/nanostructure was formed on the surface.

\subsection{Preparation of $B S A A$}

The BSAA was prepared via a sample two-step process as systematically illustrated in Figure 1. The BSAA was obtained according to the following procedure: Initially, $1.0 \mathrm{~g}$ $\mathrm{TiO}_{2} \mathrm{MPs}$ and $0.5 \mathrm{~mL}$ ammonium hydroxide ( $30 \mathrm{wt} . \%$ ) were added to $100 \mathrm{~mL}$ absolute ethanol which was heated at $45^{\circ} \mathrm{C}$ for $2 \mathrm{~h}$. Then, after reduced pressure distillation, $1.5 \mathrm{~mL}$ tetraethyl orthosilicate, and $3.5 \mathrm{~mL}$ HDMS, were poured into the above solution, and reacted at $45{ }^{\circ} \mathrm{C}$ for $12 \mathrm{~h}$. Tetraethyl orthosilicate was decomposed to $\mathrm{SiO}_{2}$ under alkaline conditions, which was beneficial for the $\mathrm{TiO}_{2}$ wrapped in $\mathrm{SiO}_{2}$ to form the $\mathrm{SiO}_{2}-\mathrm{TiO}_{2}$ particles.

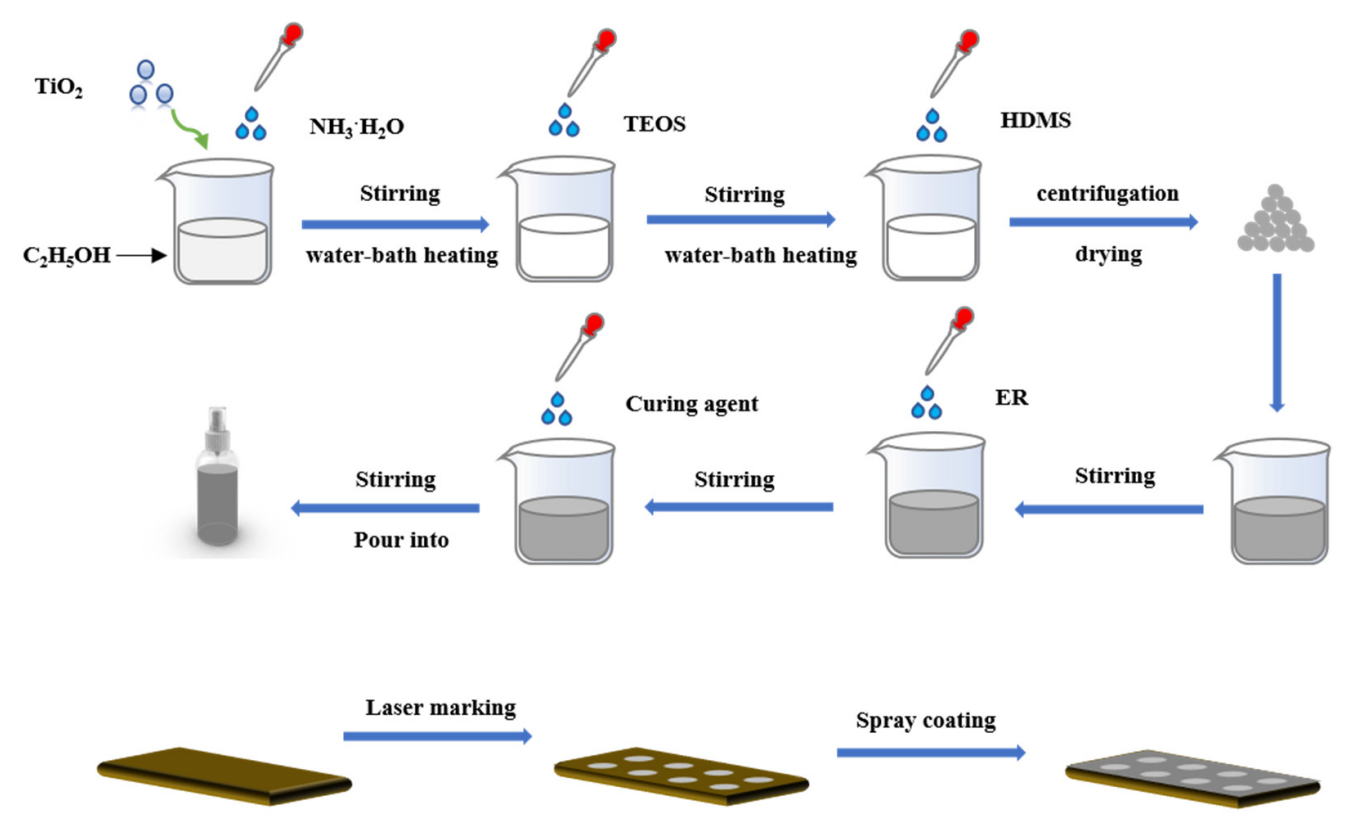

Figure 1. Preparation scheme diagram of BSAA's coating and substrate.

Moreover, the added HDMS could introduce methyl groups into newly emerging particles, which could effectively reduce the surface energy of the coating, resulting in superhydrophobicity. Afterwards, the mixture was centrifuged four times at 8000 RPM for $15 \mathrm{~min}$ each time and dried at $100{ }^{\circ} \mathrm{C}$ for three hours. The suspension was prepared as follows. An amount of $1.0 \mathrm{~g}$ grinded sediments was dispersed in $50 \mathrm{~mL}$ absolute ethanol by magnetic stirring for $10 \mathrm{~min}$. Next, a transparent ER $(0.6 \mathrm{~g})$ solution was added into the as-prepared solution dropwise at room temperature, stirred for $10 \mathrm{~min}$, and then the curing agent $(0.2 \mathrm{~g})$ was added. Subsequently, the suspension was sprayed three times on the aluminium substrate with the microstructure by an atomizer with a $1 \mathrm{~mm}$ diameter. The working distance between the atomizer orifice and the aluminium substrate was about $15 \mathrm{~cm}$ in this study. Finally, the sample was placed horizontally and dried at $60{ }^{\circ} \mathrm{C}$ for $120 \mathrm{~min}$. The BSAA was successfully attained. Furthermore, the similar characteristics between the diving bell spider and the BSAA design were investigated and distinctly revealed, as shown in Figure S1. 


\subsection{Analysis and Testing Methods}

\subsubsection{Mechanical Abrasion Durability Test}

Sandpaper (600 grid) with a standard weight (100 g) was used to abrade the coating [43-46]. The abrasion durability test was performed by pulling the sample back and forth, maintaining a distance of $10 \mathrm{~cm}$ above the horizontal sandpaper; this is a complete cycle test.

\subsubsection{Chemical Durability Test}

The chemical durability of the coating surface was tested using droplets with different acidity and alkalinity. Sodium hydroxide and hydrogen chloride were used to prepare the solution with a $\mathrm{pH}$ of 1-14. A contact angle measuring instrument (JC2000A, Biolin Scientific, Espoo, Finland) was used to observe this.

\subsubsection{Underwater Bubble Adsorption Test}

The bubble adsorption test was measured using an artificial bubble, utilizing a highspeed camera (V5.1, Phantom, New York City, NY, USA) which could capture the rising bubble. The air bubbles were released from $10 \mathrm{~cm}$ below the substrate.

\subsubsection{Other Characterizations}

The surface 3D morphology and roughness were observed by the Ultra-Depth ThreeDimensional Microscope (VHX-6000, KEYENCE, Tokyo, Japan).

The element distribution and morphology were analyzed by an Energy Dispersive Spectrometer (EDS) and Scanning Electron Microscope (SEM, JEOL Ltd., Tokyo, Japan).

\section{Results and Discussion}

\subsection{Surface Chemical Composition and Morphology}

An Ultra-Depth Three-Dimensional Microscope was used to observe the changes before and after spraying on the surface of the aluminium alloy substrate with microstructures. Figure 2a showed that the pits on the surface of the aluminium substrate were evenly distributed, with a diameter of about $100 \mu \mathrm{m}$ and a spacing of about $100 \mu \mathrm{m}$. Around each pit, there were residues in the shape of a volcanic pile formed by laser etching. This was also an important reason for the formation of the micro/nanostructure of the BSAA. The pits with an average depth of $60 \mu \mathrm{m}$ also left plenty of room for trapping bubbles. After spraying the coating, it could be seen from Figure $2 b$ that the surface of the aluminium substrate was uniformly covered by the coating. The coating covered the gap between the pits and the surrounding volcanic pile structure, leaving a circular hole in the middle, which establishes the foundation for the formation of a gas film on the surface of the subsequent sample. Morphology was observed using the Scanning Electron Microscope on the surface coating in Figure 2c,d. The layered structure was composed of $\mathrm{TiO}_{2} \mathrm{MPs}$ and $\mathrm{SiO}_{2} \mathrm{NPs}$. This could store a large amount of air in the micro/nanostructures, and the water droplets were suspended on the surface with the composite micro/nanostructures. The air prevented the water droplets from penetrating the solid surface and greatly reduced the contact area between the water droplets and the BSAA surface. As a result, the adhesion of the BSAA surface decreased, the dynamic contact angle appeared to be hysteresis, and the surface wettability presented a Cassie state with superhydrophobic properties. The roughness of the double-layer played a key role in the superhydrophobic properties of the coating. Figure $2 \mathrm{e}, \mathrm{f}$ showed that the coating was mainly composed of $\mathrm{C}, \mathrm{O}, \mathrm{Al}, \mathrm{Si}$, and $\mathrm{Ti}$ elements, and the content of $\mathrm{O}, \mathrm{Si}$ and Ti elements on the surface increased significantly. It could be seen from the content and composition of each element that the surface had been covered by low-surface energy materials. 

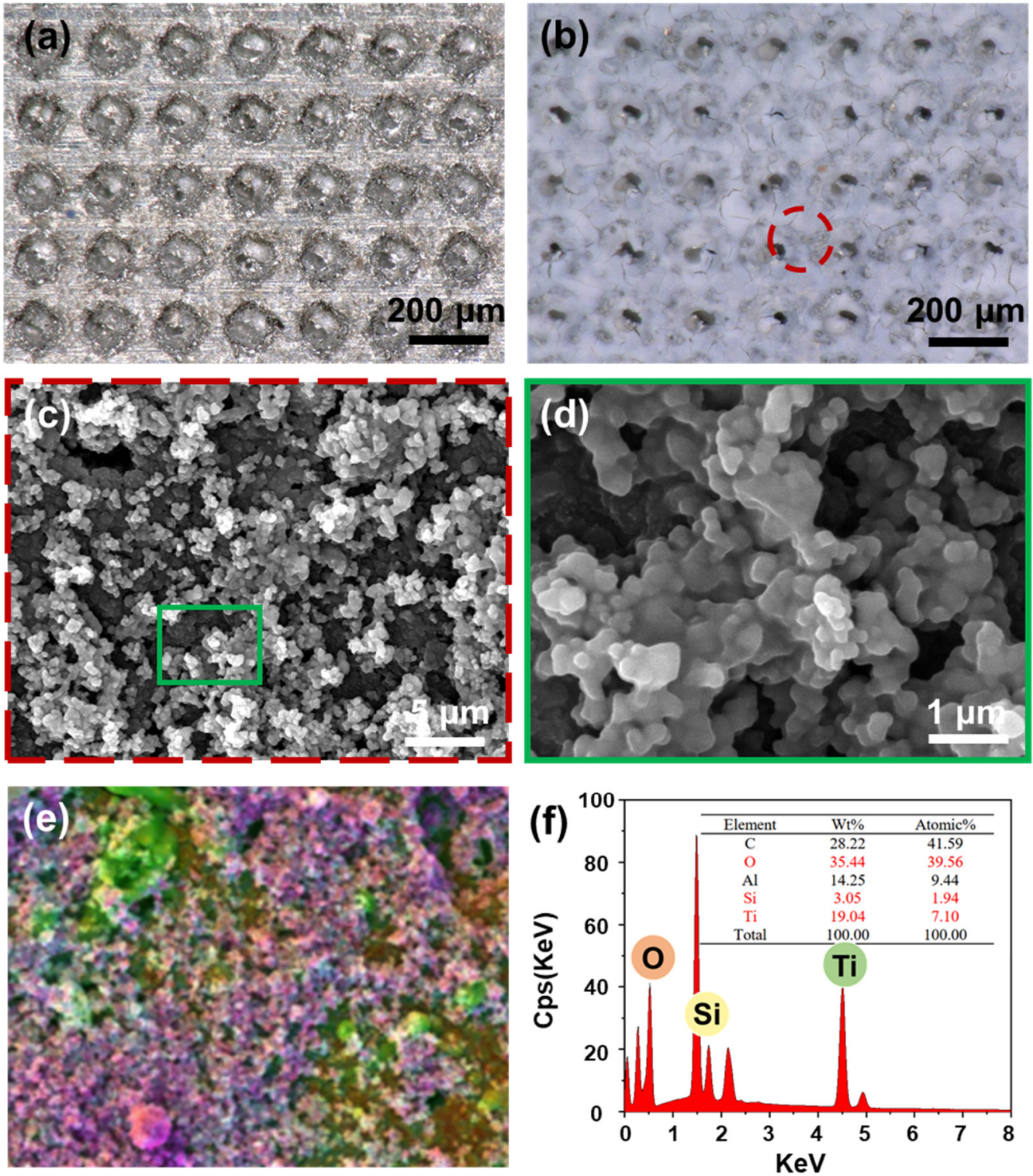

Figure 2. Micromorphology and element distribution of BSAA. (a) Original morphology of aluminium alloy substrate. (b) Morphology of bionic superhydrophobic coating. (c) SEM image of the coating surface at low magnification. (d) SEM image of the coating surface under high magnification. (e) EDS image of element distribution on the surface of BSAA. (f) Distribution and proportion of elements on the surface of BSAA.

\subsection{Non-Wetting Mechanism}

Figure 3a showed that the surface of the aluminium alloy had a distinct microstructure. The reason for the formation of these intricate micro/nanostructures was as follows: when the laser beam began to contact the material, the surface began to produce ablation, which was accompanied by the occurrence of an interference process. After applying laser energy, the molten metal could be modulated into energy stripes at the interface between the air and the substrate, and deposited on the metal surface, making the surface area of the molten metal periodic. In this process, due to the uneven energy distribution, the surface also formed part of the smaller nanostructure, and finally showed a periodic surface micro/nanocomposite structure surface. The micro/nano double-layer hierarchical structure on the prepared surface provided good conditions for the formation of a gas membrane when the aluminium alloy surface contacted the aqueous medium. In the Cassie-Baxter wetting model, it was assumed that the liquid droplet only contacted with 
the microstructure on the solid surface, that is, there was a small amount of air between the liquid and the solid, so that the liquid was suspended on the solid and air surface, as shown in Figure 3b. The characteristic contact angle in this state could be expressed by the following formula:

$$
\cos \theta_{r}=f_{1} \cos \theta_{0}-f_{2}
$$
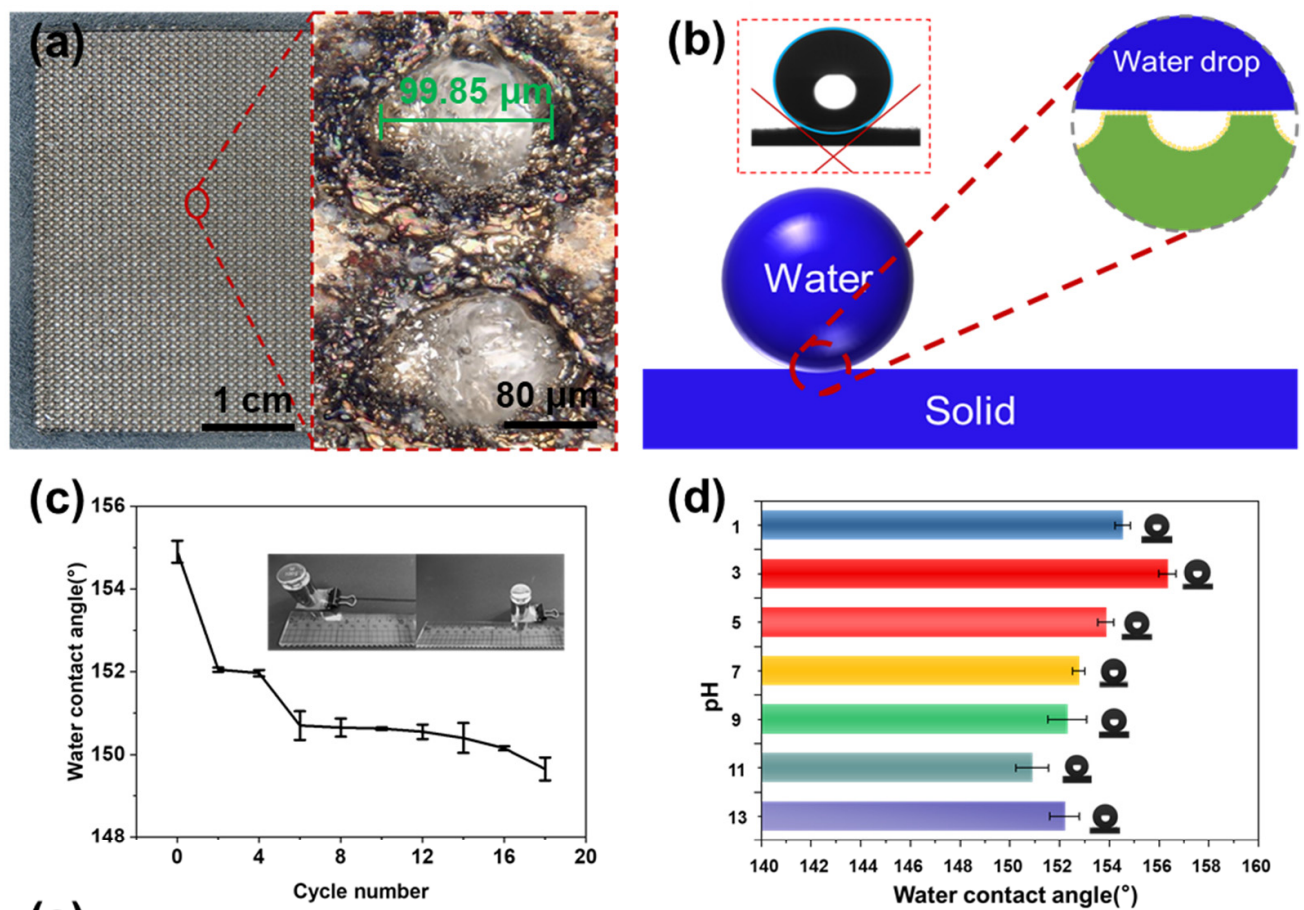

(e)
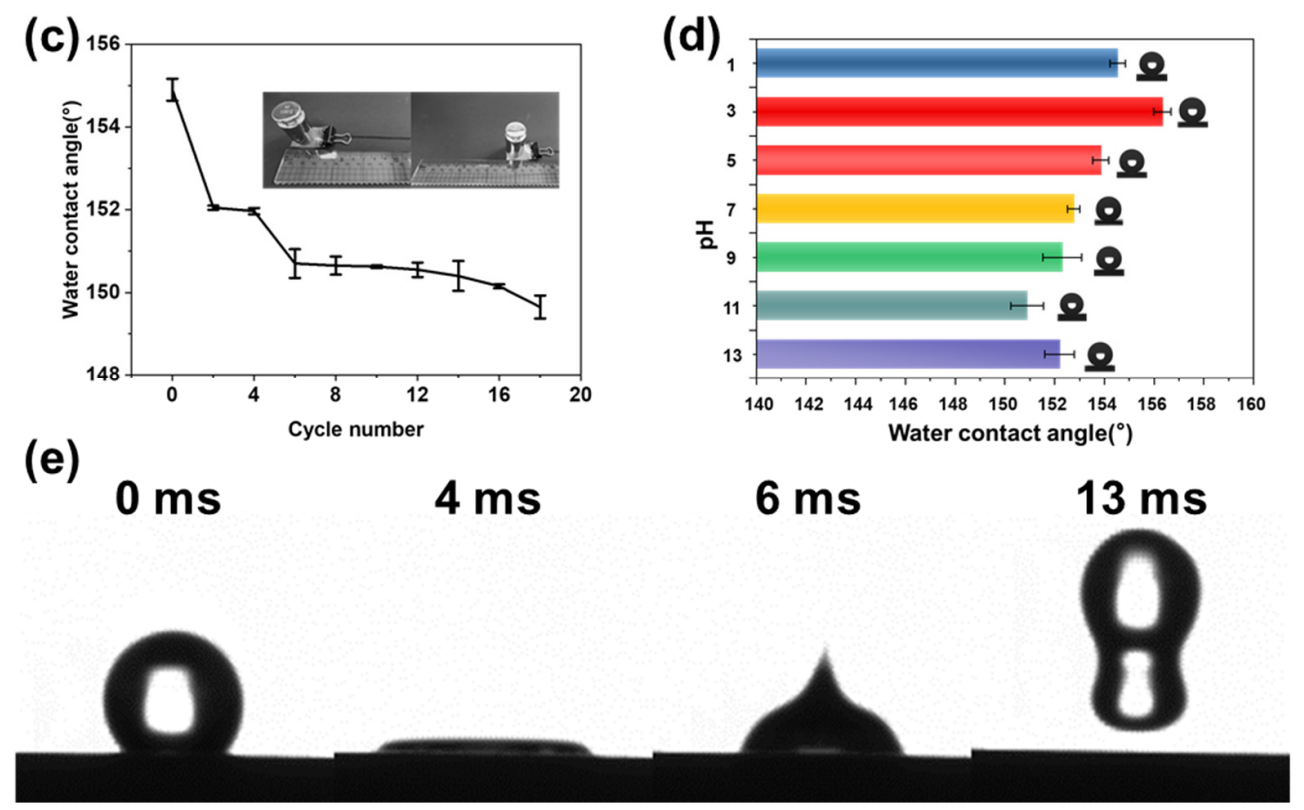

Figure 3. Superhydrophobic mechanism and characterizations of water-repellent performance. (a) Morphology of the substrate of BSAA with microcavity arrays prepared by the method of laser marking. (b) Hydrophobic mechanism diagrams of the BSAA. (c) Results of mechanical stability test. (d) Results of chemical stability test. (e) Water-bounce dynamics on BSAA.

$f_{1}$ indicates the ratio of the contact area between the droplet and the solid surface to the total contact area of the droplet. $f_{2}$ indicates the ratio of the contact area between the droplet and air to the total contact area of the drop. $\theta_{r}$ represents the contact angle of the drop on the surface of the biomimetic metal aluminium $\left(153.79^{\circ}\right) . \theta_{0}$ indicates the contact angle of the drop on the smooth unprocessed surface (the surface contact angle of aluminium is $65.43^{\circ}$ ). Since $f_{1}+f_{2}=1, f_{1}=0.0727$ was obtained by calculation. The magnitude of this value indicated the fraction of the contact area between the liquid and the solid surface, that is, the solid surface at this time had less contact with water droplets. It could be seen from the calculation results that only $7.27 \%$ of the area of the water droplets was in contact with the bionic surface of the aluminium alloy, while the remaining area $(92.73 \%)$ of the water droplets was in contact with the air. The results also further indicated that the micro/nanostructure prepared on the surface of the aluminium alloy substrate played a very important role in the superhydrophobic properties. 


\subsection{Mechanical Abrasion Durability, Chemical Durability and Low Adhesion of the Coating}

Mechanical abrasion durability had always been one of the key issues faced by superhydrophobic materials. This work solved this problem by using epoxy resin (ER) as an adhesive. Figure $3 \mathrm{c}$ showed that during the entire mechanical abrasion durability experiment, the water contact angle (WCA) decreased significantly in the first six cycles. In the following ten experiments, the WCA on the sample surfaces were without obvious changes. This was because the newly exposed $\mathrm{SiO}_{2}-\mathrm{TiO}_{2}$ and $\mathrm{ER}$ restructured the roughness after the surface was abraded to achieve superhydrophobicity. After the test was performed 16 times, the superhydrophobic properties of the coating surface were lost.

The chemical durability of the coating surface was tested in Figure 3d. By configuring solutions with different $\mathrm{pHs}$ varying from 1 to 14 , a $4 \mu \mathrm{L}$ droplet with a different $\mathrm{pH}$ was added to samples for $10 \mathrm{~min}$ each time, and each test was repeated at least three times. With the help of a drop shape analysis instrument, the BSAA exhibited favorable superhydrophobicity with the contact angle of BSAA varying from $150.9 \pm 0.66^{\circ}$ to $156.3 \pm 0.35^{\circ}$. This result confirmed that no matter how the $\mathrm{pH}$ value of the aqueous solution changed, the surface of BSAA could ensure the stable existence of superhydrophobic properties, which revealed that the $\mathrm{pH}$ value of the aqueous solution did not have much influence on the superhydrophobic surface of BSAA. In other words, it was feasible to select aluminium alloy as a functional material to prepare the superhydrophobic surface. Furthermore, as shown in Figure S2, the changes in the contact angle at a fixed time were analyzed, according to the $\mathrm{pH}$ and the time taken to decrease the contact angle by 10 degrees for each $\mathrm{pH}$. In addition, the changes in the contact angle of the BSAA immersed in salt solution were also investigated. The concentration of the $\mathrm{NaCl}$ in water is similar to that of the South China Sea.

In order to check the liquid wetting behavior of the BSAA surface, a high-speed camera system was used to record the rebound dynamics of the water droplets. The diffusion process of droplets is shown in Figure 3e. When using a pipette to drop $5 \mathrm{ul}$ of water droplets from $5 \mathrm{~cm}$ of height onto the surface of the BSAA, the whole process could be observed by utilizing the high-speed camera. The water droplet spread immediately after contacting the surface, and the water film spread to its maximum at $4 \mathrm{~ms}$, then the droplet shrank rapidly and was completely removed from the surface at $13 \mathrm{~ms}$. This showed that the BSAA had excellent superhydrophobicity and low adhesion.

Figure S3a,b demonstrated the morphology of the aluminium before and after the mechanical abrasion durability test. Figure S3c,d shown the morphology of the BSAA before and after the mechanical abrasion durability test. It could be concluded that the aluminium had a much more severe abrasion after the mechanical abrasion durability test compared with the BSAA case. In addition, Figure S4a,b shown the morphology of the BSAA before and after the chemical stability test. Figure S4c,d demonstrated the morphology of the aluminium before and after the chemical stability test. It could be found that the aluminium had a much more severe corrosion after the chemical stability test compared with the BSAA case.

\subsection{Characteristics of Underwater Bubbles Adsorption}

To study the gas membrane manipulation below the aluminium-based superhydrophobic surface in a water environment, the control state of the air bubbles beneath the aluminium-based superhydrophobic surface was observed by a self-built high-speed camera system in Figure 4. 


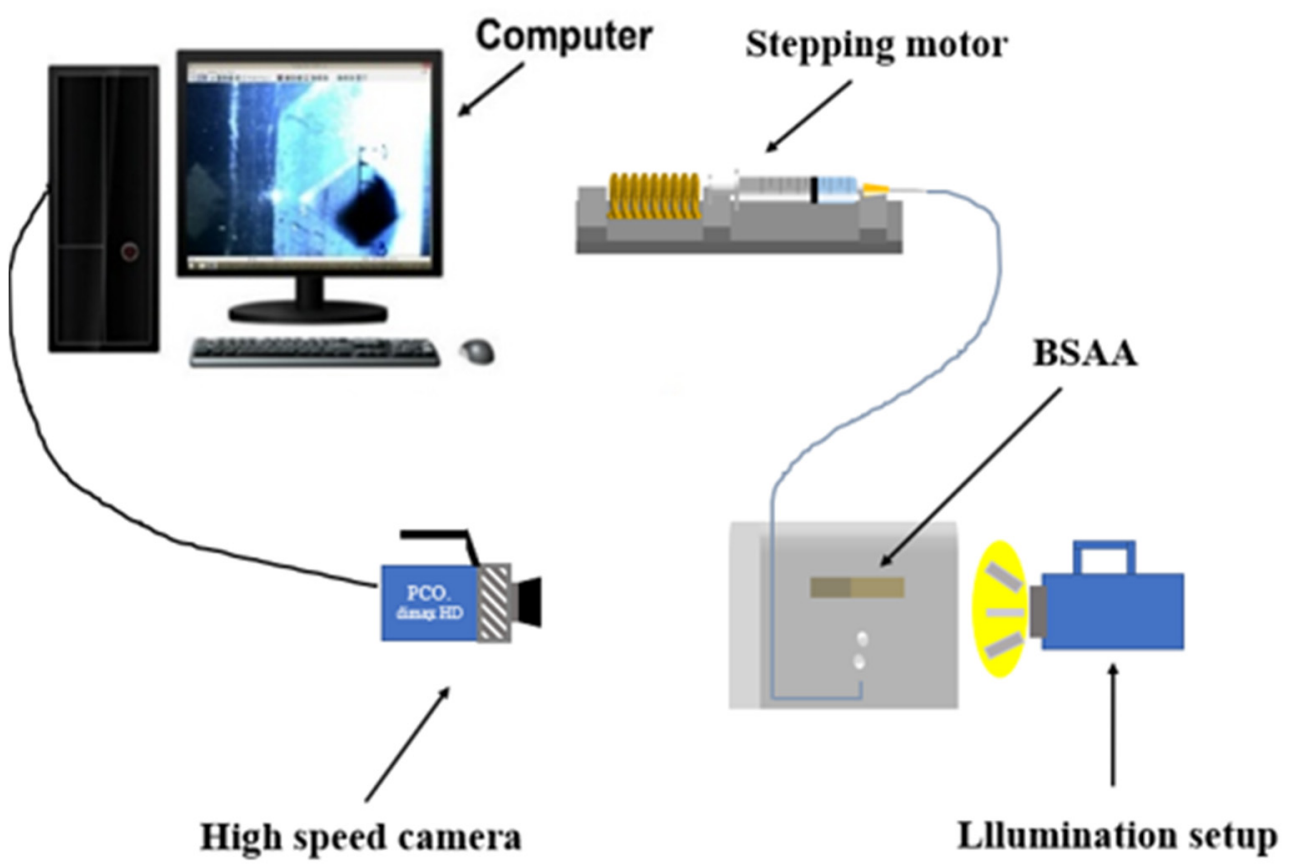

Figure 4. Self-built high-speed camera system for the observation of the capturing behavior of the BSAA for the air bubbles.

The system inputs bubbles regularly through a stepper motor. Figure 5a illustrates a smooth hydrophilic aluminium-based surface underwater, a WCA about $65^{\circ}$ in the air. It could be seen from Figure 5 a and Video S1 show that the smooth aluminium alloy surface exhibited resists bubbles' properties in an underwater environment. The bubbles appear as a quasi-spherical shape on the surface, with a contact angle of about $152^{\circ}$, and they could easily roll out of the surface. The bubble in the figure bounced and rolled quickly after hitting the aluminium alloy surface, which showed the resistance of the aluminium base to bubbles under water. Figure $5 b$ and Video S2 displayed the state of the motion of the bubbles on the surface under different time conditions in an underwater environment. As observed in the Figures, when a bubble contacted the surface, the bubble would spread out rapidly within $5 \mathrm{~ms}$. Until $15 \mathrm{~ms}$, it become a stable state, and its contact angle reached $26^{\circ}$. BSAA showed the characteristics of affinity to bubbles. The main reason for the above phenomenon is the difference between the solid surface energy, and the liquid and the gas surface energy.

It is worth noting that two bubbles were stacked together to form a continuous gas membrane in Figure 5c. The pits on the surface of the aluminium base could effectively trap the bubbles and make the bubbles stably exist on the surface of the sample for a long time. We can say it reflects that multiple bubbles could form a gas membrane on the surface of the sample, instead of being independent of each other. Figure $5 \mathrm{~d}$ showed the state of the sample after absorbing bubbles under water. A single bubble could maintain on the superhydrophobic surface for more than $8 \mathrm{~s}$ under water. When air bubbles were continuously inputted on to the superhydrophobic surface, at this time, a thin protective film of water could be formed on the surface, which was equivalent to that of the gas film wrapped in the entire aluminium-based sample, and created a lubricating effect on the surface. Therefore, the prepared superhydrophobic sample could effectively trap air bubbles in a water environment, which was very similar to the process that allowed the diving bell spider to breathe under water. Controlling the behavior of bubbles on solid surfaces in a liquid environment has broad application prospects, such as new medical equipment, microfluidic chips, and gas separation and collection in water. It also provides a reference experience for the promotion and application of underwater drag reduction. 

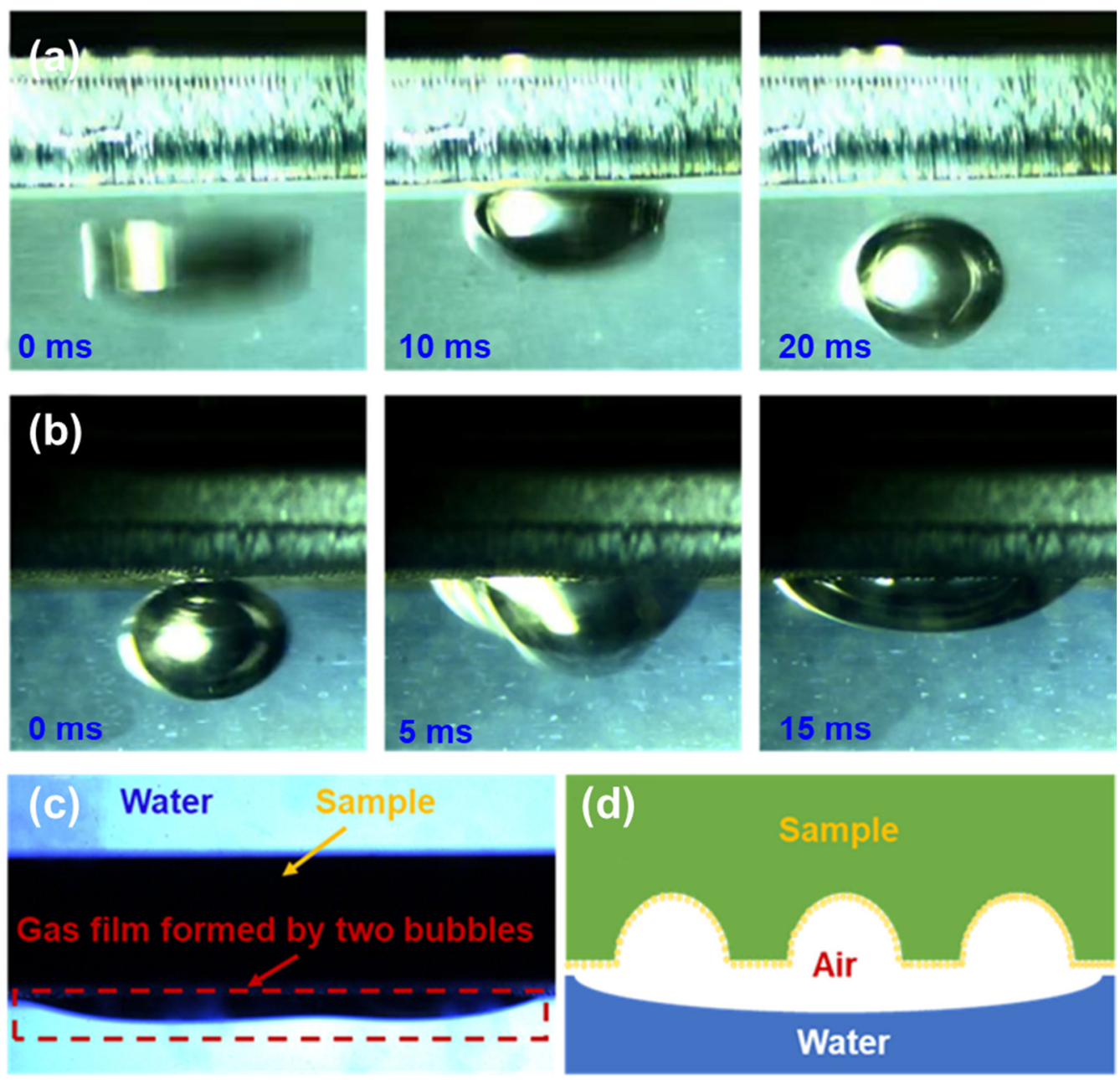

Figure 5. Study on the capturing behaviors of the BSAA for the underwater bubbles and the related mechanism. (a) Capturing behaviors for an underwater bubble on the surface of smooth aluminium alloy. (b) Capturing behaviors for an underwater bubble on the surface of BSAA. (c) Capturing behaviors for two underwater bubbles on the surface of BSAA. (d) Capturing principle of the BSAA for underwater bubbles.

\subsection{Application Prospect of BSAA}

This work can be applied to the field of ship navigation in the future. When a ship is sailing in the sea, the adsorption of a large number of marine anaerobic organisms on the navigation body can cause the hull to corrode and greatly reduced the service life. In this work, the gas membrane wrapped around the hull, which was due to the numerous multi-structures of superhydrophobic pits trapping air bubbles. Among them, the volcanic pile-structure of BSAA plays an important role in capturing the bubbles, as shown in Figure 6a. The gas membrane attached to this layer of the ship isolated the hull from the sea directly. As a result, the resistance was changed from solid-liquid contact to solid-gas contact, which greatly reduced the resistance of the ship. The adsorption of a large number of marine anaerobic organisms on the navigation body would cause the hull to corrode and greatly reduced the service life. in other words, in Figure $6 \mathrm{~b}$, the existence of the gas membrane prevented anaerobic organisms from contacting the hull, thus preventing the hull from corroding. This research provided important research ideas for the future multi-functional drag reduction of sailing bodies, as well as a novel direction for the drag reduction of underwater vehicles. 


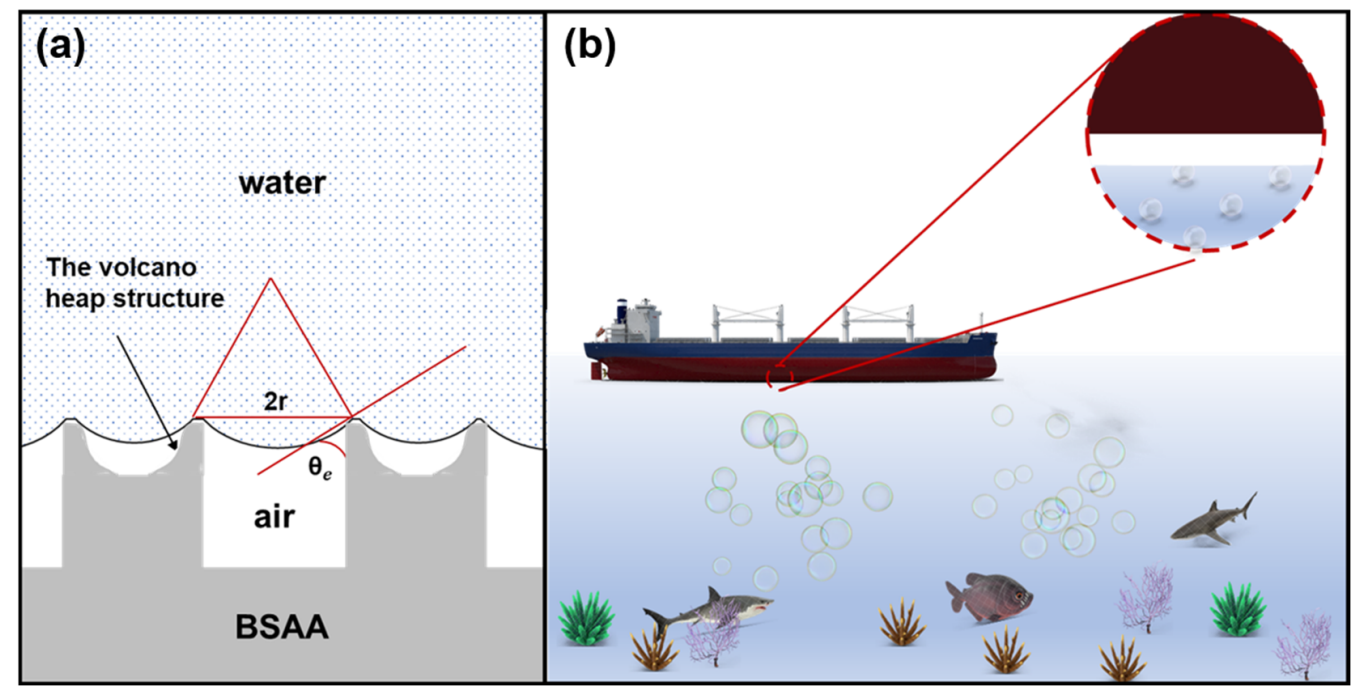

Figure 6. Mechanism of physicochemical durability of BSAA and the applications. (a) Schematic diagram of gas film on BSAA surface. (b) Schematic diagram of future application 4.

\section{Conclusions}

Inspired by the diving bell spider, a biomimetic superhydrophobic coating was prepared by a two-step synthesis method. The mixed ER and $\mathrm{SiO}_{2}-\mathrm{TiO}_{2}$ particles were evenly distributed on the surface of the aluminium. The prepared BASS possessed excellent superhydrophobic properties. The WCA of BASS has a high static contact angle value (about $153^{\circ}$ ). Furthermore, mechanical abrasion durability and chemical durability have also been proved. BSAA could withstand 16 times on the mechanical abrasion durability test and have chemical durability in all $\mathrm{pH}$ ranges. Under high-speed camera observation, the BSAA exhibits excellent low adhesion characteristics, which provided an important prerequisite for underwater navigation without contamination of aquatic organisms. Furthermore, the BSAA shows a surprising bubble-capture capacity underwater, which could absorb bubbles quickly floating in the water in $5 \mathrm{~ms}$. It is proposed that it would effectively reduce resistance and avoid attachment of the anaerobic organisms, due to the existence of the gas membrane when sailing. Therefore, we predict that the BSAA with excellent physicochemical properties may enlighten more scientists and engineers to focus on mimicking natural structures in a simple and effective method, and to devise more ingenious engineering products with desirable functionalities in the future.

Supplementary Materials: The following are available online at https: / www.mdpi.com/article / 10.3390/coatings11101146/s1, Figure S1: SEM images of the water spider [40] and BSAA. (a) SEM image of the abdomen of water spider. (b) SEM image of the microstructures on the abdominal surface of water spider. (c) SEM image of the BSAA. (d) SEM image of the single hole. (e) SEM image of the microstructures on the BSAA surface, Figure S2: (a) A simple diagram of the drop shape analyzer and the sliding angle of the BSAA. (b) The time required to change the WCAs by 5 degrees. (c) WCAs of the BSAA changing with the time in different $\mathrm{pH}$. (d) WCAs of the BSAA changing with the time in different $\mathrm{NaCl}$ solutions, Figure S3: Surface morphology of the aluminium alloy and BSAA before and after the mechanical abrasion test. (a) Surface morphology of the aluminium alloy before the mechanical abrasion test. (b) Surface morphology of the aluminium alloy after the mechanical abrasion test. (c) Surface morphology of the BSAA before the mechanical abrasion test. (d) Surface morphology of the BSAA after the mechanical abrasion test, Figure S4: Surface morphology of BSAA and aluminium alloy before and after chemical stability test. (a) Surface morphology of BSAA before chemical stability test. (b) Surface morphology of BSAA after chemical stability test. (c) Surface morphology of aluminium alloy before chemical stability test. (d) Surface morphology of aluminium alloy after chemical stability test, Video S1: Capturing behaviors for an underwater bubble on the surface of smooth aluminum alloy, Video S2: Capturing behaviors for an underwater bubble on the surface of BSAA. 
Author Contributions: Conceptualization, B.L. and S.N.; Data curation, B.L. and S.N.; Funding acquisition, B.L., S.N. and Z.H.; Investigation, Z.Q., Y.S., D.C. and D.L.; Methodology, Y.C., Z.W., B.L. and S.N.; Project administration, Z.H.; Resources, Z.H.; Supervision, Z.H. and L.R.; Validation, Y.C., L.Z., J.Z. and Z.M.; Writing-original draft, Y.C.; Writing-review and editing, B.L. and S.N. All authors have read and agreed to the published version of the manuscript.

Funding: This work was supported by the National Key Research and Development Program of China (No. 2018YFA0703300), the Foundation for Innovative Research Groups of the National Natural Science Foundation of China (No. 52021003), the National Natural Science Foundation of China (Nos. 51835006, 51875244, U19A20103, 52105301 and 52105298), the Jilin University Science and Technology Innovative Research Team (2020TD-03), the Natural Science Foundation of Jilin Province (No. 20200201232JC), China Postdoctoral Science Foundation (Nos. 2021TQ0121, 2021M691205 and 2020M670844), National Postdoctoral Program for Innovative Talents (BX20190139), Graduate Student Innovation Fund of Jilin University (No. 2016020), the Ph.D. Interdisciplinary Research Funding Scheme of Jilin University (No. 101832020DJX050), Open Innovation Project of the 55th Research Institute of NORINCO GROUP, and Joint Fund for Independent Innovation of Future Laboratory of the 2nd Research Institute of CASIC.

Institutional Review Board Statement: Not applicable.

Informed Consent Statement: Not applicable.

Data Availability Statement: The data that support the findings of this study are available from the corresponding author, upon reasonable request.

Conflicts of Interest: The authors declare that they have no known competing financial interests or personal relationships that could have appeared to influence the work reported in this paper.

\section{References}

1. Ceccio, S.L. Friction drag reduction of external flows with bubble and gas injection. Annu. Rev. Fluid Mech. 2010, 42, 183-203. [CrossRef]

2. White, C.M.; Mungal, M.G. Mechanics and prediction of turbulent drag reduction with polymer additives. Annu. Rev. Fluid Mech. 2008, 40, 235-256. [CrossRef]

3. Rothstein, J.P. Slip on superhydrophobic surfaces. Annu. Rev. Fluid Mech. 2010, 42, 89-109. [CrossRef]

4. Chen, X.; Wang, P.; Zhang, D. Designing a Superhydrophobic Surface for Enhanced Atmospheric Corrosion Resistance Based on Coalescence-Induced Droplet Jumping Behavior. ACS Appl. Mater. Interfaces 2019, 11, 38276-38284. [CrossRef] [PubMed]

5. Sebastian, D.; Yao, C.-W.; Nipa, L.; Lian, I.; Twu, G. Corrosion Behavior and Mechanical Properties of a Nanocomposite Superhydrophobic Coating. Coatings 2021, 11, 652. [CrossRef]

6. Sebastian, D.; Yao, C.W.; Lian, I. Mechanical durability of engineered superhydrophobic surfaces for anti-corrosion. Coatings 2018, 8, 162. [CrossRef]

7. Du, C.; He, X.; Tian, F.; Bai, X.; Yuan, C. Preparation of Superhydrophobic Steel Surfaces with. Coatings 2019, 9, 398. [CrossRef]

8. Vazirinasab, E.; Jafari, R.; Momen, G. Application of superhydrophobic coatings as a corrosion barrier: A review. Surf. Coat. Technol. 2018, 341, 40-56. [CrossRef]

9. Mukherjee, S.; Martínez-González, J.A.; Stallard, C.P.; Dowling, D.P.; Gowen, A.A. Can attenuated total internal reflection-fourier transform infrared be used to understand the interaction between polymers and water? A hyperspectral imaging study. J. Spectr. Imaging 2017, 6, 1-13.

10. Mukherjee, S.; Martínez-González, J.A.; Dowling, D.P.; Gowen, A.A. Predictive modelling of the water contact angle of surfaces using attenuated total reflection-Fourier transform infrared (ATR-FTIR) chemical imaging and partial least squares regression (PLSR). Analyst 2018, 143, 3729-3740. [CrossRef]

11. Mukherjee, S.; Martinez-Gonzalez, J.A.; Gowen, A.A. Feasibility of attenuated total reflection-fourier transform infrared (ATRFTIR) chemical imaging and partial least squares regression (PLSR) to predict protein adhesion on polymeric surfaces. Analyst 2019, 144, 1535-1545. [CrossRef] [PubMed]

12. Zhang, X.; Shi, F.; Niu, J.; Jiang, Y.; Wang, Z. Superhydrophobic surfaces: From structural control to functional application. J. Mater. Chem. 2008, 18, 621-633. [CrossRef]

13. Yin, L.; Yang, J.; Tang, Y.; Chen, L.; Liu, C.; Tang, H.; Li, C. Mechanical durability of superhydrophobic and oleophobic copper meshes. Appl. Surf. Sci. 2014, 316, 259-263. [CrossRef]

14. Yao, C.-W.; Sebastian, D.; Lian, I.; Günaydın-Şen, Ö.; Clarke, R.; Clayton, K.; Chen, C.-Y.; Kharel, K.; Chen, Y.; Li, Q. Corrosion Resistance and Durability of Superhydrophobic Copper Surface in Corrosive NaCl Aqueous Solution. Coatings 2018, 8, 70. [CrossRef]

15. Bai, Y.; Zhang, H.; Shao, Y.; Zhang, H.; Zhu, J. Recent progresses of superhydrophobic coatings in different application fields: An overview. Coatings 2021, 11, 116. [CrossRef] 
16. Ebert, D.; Bhushan, B. Transparent, superhydrophobic, and wear-resistant coatings on glass and polymer substrates using $\mathrm{SiO}_{2}$, $\mathrm{ZnO}$, and ITO nanoparticles. Langmuir 2012, 28, 11391-11399. [CrossRef]

17. Zhuang, A.; Yang, L.; Liao, R.; Guo, C.; Zuo, Z.; Yuan, Y. A simple method to make mechanically robust, adhesive and superhydrophobic surface based on epoxy resin. J. Coat. Technol. Res. 2015, 12, 609-615. [CrossRef]

18. Jena, G.; Thinaharan, C.; George, R.P.; Philip, J. Robust nickel-reduced graphene oxide-myristic acid superhydrophobic coating on carbon steel using electrochemical codeposition and its corrosion resistance. Surf. Coat. Technol. 2020, 397, 125942. [CrossRef]

19. Wang, D.; Sun, Q.; Hokkanen, M.J.; Zhang, C.; Lin, F.Y.; Liu, Q.; Zhu, S.P.; Zhou, T.; Chang, Q.; He, B.; et al. Design of robust superhydrophobic surfaces. Nature 2020, 582, 55-59. [CrossRef]

20. Lou, X.; Huang, Y.; Yang, X.; Zhu, H.; Heng, L.; Xia, F. External Stimuli Responsive Liquid-Infused Surfaces Switching between Slippery and Nonslippery States: Fabrications and Applications. Adv. Funct. Mater. 2020, 30, 1901130. [CrossRef]

21. Zhang, S.; Zhang, J.; Zhu, B.; Niu, S.; Han, Z.; Ren, L. Progress in Bio-inspired Anti-solid Particle Erosion Materials: Learning from Nature but Going beyond Nature. Chin. J. Mech. Eng. 2020, 33, 1-27. [CrossRef]

22. Kobina Sam, E.; Kobina Sam, D.; Lv, X.; Liu, B.; Xiao, X.; Gong, S.; Yu, W.; Chen, J.; Liu, J. Recent development in the fabrication of self-healing superhydrophobic surfaces. Chem. Eng. J. 2019, 373, 531-546. [CrossRef]

23. Emelyanenko, K.A.; Emelyanenko, A.M.; Boinovich, L.B. Water and ice adhesion to solid surfaces: Common and specific, the impact of temperature and surface wettability. Coatings 2020, 10, 648. [CrossRef]

24. Xiang, T.; Lv, Z.; Wei, F.; Liu, J.; Dong, W.; Li, C.; Zhao, Y.; Chen, D. Superhydrophobic Civil Engineering Materials: A Review from Recent Developments. Coatings 2019, 9, 753. [CrossRef]

25. Xue, C.-H.; Jia, S.-T.; Zhang, J.; Ma, J.-Z. Large-area fabrication of superhydrophobic surfaces for practical applications: An overview. Sci. Technol. Adv. Mater. 2010, 11, 033002. [CrossRef] [PubMed]

26. Bao, W.; Liang, D.; Zhang, M.; Jiao, Y.; Wang, L.; Cai, L.; Li, J. Durable, high conductivity, superhydrophobicity bamboo timber surface for nanoimprint stamps. Prog. Nat. Sci. Mater. Int. 2017, 27, 669-673. [CrossRef]

27. Liang, Z.; Zhou, Z.; Dong, B.; Wang, S. Fabrication of Superhydrophobic and UV-Resistant Silk Fabrics with Laundering Durability and Chemical Stabilities. Coatings 2020, 10, 349. [CrossRef]

28. Ma, G.; Zheng, H.; Chang, S.; Wang, S. Wettability Analysis and Design of Micro-nanostructured Superhydrophobic Surface. Acta Chim. Sin. 2019, 77, 269-277. [CrossRef]

29. Zhai, L.; Cebeci, F.C.; Cohen, R.E.; Rubner, M.F. Stable superhydrophobic coatings from polyelectrolyte multilayers. Nano Lett. 2004, 4, 1349-1353. [CrossRef]

30. Liu, K.; Li, Z.; Wang, W.; Jiang, L. Facile creation of bio-inspired superhydrophobic Ce-based metallic glass surfaces. Appl. Phys. Lett. 2011, 99. [CrossRef]

31. Chi, J.; Zhang, X.; Wang, Y.; Shao, C.; Shang, L.; Zhao, Y. Bio-inspired wettability patterns for biomedical applications. Mater. Horiz. 2021, 8, 124-144. [CrossRef]

32. Liu, K.; Jiang, L. Bio-inspired design of multiscale structures for function integration. Nano Today 2011, 6, 155-175. [CrossRef]

33. Sun, N.; Qin, S.; Wu, J.; Cong, C.; Qiao, Y.; Zhou, Q. Bio-Inspired superhydrophobic polyphenylene sulfide/polytetrafluoroethylene coatings with high performance. J. Nanosci. Nanotechnol. 2012, 12, 7222-7225. [CrossRef] [PubMed]

34. Han, Z.; Feng, X.; Guo, Z.; Niu, S.; Ren, L. Flourishing Bioinspired Antifogging Materials with Superwettability: Progresses and Challenges. Adv. Mater. 2018, 30, 1704652. [CrossRef]

35. Han, Z.; Mu, Z.; Yin, W.; Li, W.; Niu, S.; Zhang, J.; Ren, L. Biomimetic multifunctional surfaces inspired from animals. Adv. Colloid Interface Sci. 2016, 234, 27-50. [CrossRef] [PubMed]

36. Peng, J.; Cheng, Q. High-Performance Nanocomposites Inspired by Nature. Adv. Mater. 2017, 29, 1702959. [CrossRef] [PubMed]

37. Zhang, D.; Zhang, W.; Gu, J.; Fan, T.; Liu, Q.; Su, H.; Zhu, S. Inspiration from butterfly and moth wing scales: Characterization, modeling, and fabrication. Prog. Mater. Sci. 2015, 68, 67-96. [CrossRef]

38. Gu, J.; Zhang, W.; Su, H.; Fan, T.; Zhu, S.; Liu, Q.; Zhang, D. Morphology genetic materials templated from natural species. Adv. Mater. 2015, 27, 464-478. [CrossRef]

39. Wu, L.; He, J.; Shang, W.; Deng, T.; Gu, J.; Su, H.; Liu, Q.; Zhang, W.; Zhang, D. Optical Functional Materials Inspired by Biology. Adv. Opt. Mater. 2016, 4, 195-224. [CrossRef]

40. Neumann, D.; Woermann, D. Stability of the volume of air trapped on the abdomen of the water spider Argyroneta aquatica. Springerplus 2013, 2, 694. [CrossRef]

41. Neumann, D.; Kureck, A. Composite structure of silken threads and a proteinaceous hydrogel which form the diving bell wall of the water spider agyroneta aquatica. Springerplus 2013, 2, 223. [CrossRef]

42. Hu, H.; Wen, J.; Bao, L.; Jia, L.; Song, D.; Song, B.; Pan, G.; Scaraggi, M.; Dini, D.; Xue, Q. Significant and stable drag reduction with air rings confined by alternated superhydrophobic and hydrophilic strips. Sci. Adv. 2017, 3, e1603288. [CrossRef] [PubMed]

43. Lu, Y.; Sathasivam, S.; Song, J.; Crick, C.R.; Carmalt, C.J.; Parkin, I.P. Robust self-cleaning surfaces that function when exposed to either air or oil. Science 2015, 347, 1132-1135. [CrossRef] [PubMed]

44. Hu, C.; Chen, W.; Li, T.; Ding, Y.; Yang, H.; Zhao, S.; Tsiwah, E.A.; Zhao, X.; Xie, Y. Constructing non-fluorinated porous superhydrophobic $\mathrm{SiO}_{2}$-based films with robust mechanical properties. Colloids Surf. A Physicochem. Eng. Asp. 2018, 551, 65-73. [CrossRef] 
45. Davis, A.; Surdo, S.; Caputo, G.; Bayer, I.S.; Athanassiou, A. Environmentally Benign Production of Stretchable and Robust Superhydrophobic Silicone Monoliths. ACS Appl. Mater. Interfaces 2018, 10, 2907-2917. [CrossRef] [PubMed]

46. Wang, S.; Wu, S.; Zhang, J.; Wang, T. One-step fabrication of recyclable and robust fluorine/polymer-free superhydrophobic fabrics. RSC Adv. 2017, 7, 24374-24381. [CrossRef] 